\title{
Protée
}

\section{Improviser l'autre : spontanéité et structure dans la danse expérimentale contemporaine}

\section{Susan Leigh Foster}

Volume 29, numéro 2, 2001

Danse et altérité

URI : https://id.erudit.org/iderudit/030623ar

DOI : https://doi.org/10.7202/030623ar

Aller au sommaire du numéro

Éditeur(s)

Département des arts et lettres - Université du Québec à Chicoutimi

ISSN

0300-3523 (imprimé)

1708-2307 (numérique)

Découvrir la revue

Citer cet article

Leigh Foster, S. (2001). Improviser l'autre : spontanéité et structure dans la danse expérimentale contemporaine. Protée, 29(2), 25-37.

https://doi.org/10.7202/030623ar
Résumé de l'article

Une conception de l'improvisation qui prévalait dans de nombreuses expérimentations artistiques des années soixante oppose le spontané au structuré. Cet article examine les conceptions implicites du genre et de la race que met en jeu cette image de l'improvisation, proposant de voir dans cette opposition entre le spontané et le figuré la présence implicite d'un Autre féminin et racial qui permet de découvrir la nouveauté. En examinant la façon dont l'improvisation s'est implantée dans les années soixante, j'espère contribuer à notre compréhension de l'image de l'Autre que construit la danse. J'espère aussi fournir des outils pour une critique sociale et une contestation des normes. 


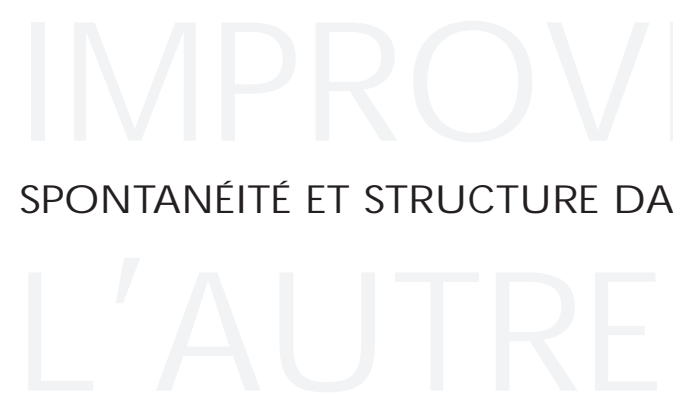

\section{IM PRO VISER L'AUTRE:}

Traduit de l'anglais par Jean-Pierre Vidal

SU SAN LEIgH FoSTER

Je me propose ici d'esquisser les contours d'une recherche sur les fondements épistémologiques de l'improvisation telle qu'elle se pratiquait, dans les années soixante, en danse et dans d'autres arts de la scène. Divers artistes œuvrant dans une grande variété de médiums envisageaient alors l'improvisation comme une stratégie permettant de révéler aux spectateurs de nouvelles façons d'être et d'agir dans le monde. L'improvisation leur offrait la possibilité de mettre l'accent plus sur le processus que sur le produit et d'insister davantage sur l'accomplissement de la performance que sur son aboutissement dans ce qu'il peut avoir de construit et de plus spectaculaire. Ils se servaient de l'improvisation pour changer radicalement les relations habituelles entre le chorégraphe et les danseurs ou entre le metteur en scène et les comédiens, en conjuguant leurs efforts pour assumer collectivement la responsabilité de l'œuvre à créer et partager la capacité d'intervenir dans sa production même. Et ils recherchaient l'improvisation comme une forme de performance plus authentique, susceptible de donner aux spectateurs un certain pouvoir en leur ménageant un accès sensiblement nouveau à la performance. Certains événements, comme les happenings et les concerts du collectif international Fluxus, ménageaient une place à la spontanéité au sein de la performance en choisissant de la réaliser dans un environnement incontrôlable ou en invitant le public à y participer de toutes sortes de façons. D'autres créateurs, comme Cage et Cunningham, s'en remettaient à une certaine indétermination pour faire naître des situations où des décisions aléatoires, conduisant à certaines suites d'actions, étaient prises juste avant ou même pendant la performance.

D'autres encore, rattachés au Living Theater et certains des chorégraphes de la Judson Church, improvisaient véritablement en prenant à chaque fois des décisions spontanées sur les actions à venir. Toutes ces expérimentations se fondaient sur le besoin de réorganiser le processus créatif afin de permettre à l'inattendu d'intervenir dans l'œuvre d'art, et sur l'urgence de mettre en évidence l'immédiateté conçue de façon suffisamment large pour inclure l'action painting de Jackson Pollock et même la poésie de Charles Olson.

L'idée dominante qui inspire nombre d'expérimentations artistiques de ce type revient à opposer le spontané au structuré. Selon cette conception, l'improvisation 
permet de se libérer de la conscience réflexive de façon à pouvoir "être" pleinement dans l'instant et se brancher ainsi sur ses impulsions les plus authentiquement spontanées. Les artistes qui adoptaient cette approche avaient accédé à l'âge adulte dans le sillage de la Seconde Guerre mondiale et des bureaucraties corporatistes qui s'étaient constituées pour y faire face, et beaucoup d'entre eux pensaient qu'en de telles circonstances un renouveau social ne pouvait venir que d'un renouvellement des facultés critiques et de la perception. L'improvisation leur paraissait constituer un véhicule essentiel de ce renouvellement des perceptions et de la sensibilité. Selon eux, en débarrassant leur esprit de toute structure préconçue et de tout automatisme, les performers et les spectateurs pourraient faire naître de nouvelles façons de percevoir le monde et de se situer par rapport à lui.

Dans son livre The Culture of Spontaneity, Daniel Belgrad soutient que ce projet esthétique, loin de refuser l'engagement politique, comme pourrait le faire croire sa comparaison avec les mouvements socialistes et communistes des années trente en particulier, mettait en jeu une nouvelle conception du politique, une conception qui se donnait pour but de renverser les régimes bureaucratiques qui donnaient leur forme à la fois au travail et aux activités de loisir. La meilleure façon de s'opposer à cette organisation corporatiste du monde consistait à susciter des événements et des stimuli parfaitement inattendus et donc porteurs de chaos. De plus, prétend Belgrad, le défi lancé par l'improvisation aux pratiques artistiques traditionnelles rendait les arts accessibles à la participation de ceux qui étaient auparavant exclus de l'univers privilégié de la création artistique:

The "open» or "heteroglossic» forms developed by the wartime avant-garde challenged the social power of American's dominant Anglo-American tradition. The aesthetic of spontaneity emphasized "honesty", "awareness", and "authenticity" over the mastery of traditional forms and techniques stressed by the established institutions of high culture. It therefore provided an alternative means to cultural authority more accessible to aspirants from immigrant, working-class, and minority backgrounds. ${ }^{1}$
Je voudrais remettre en question l'usage un peu facile, sous la plume de Belgrad, de notions comme «l'honnêteté» ou «l'authenticité» ainsi que la capacité qu'il leur attribue de donner la chance aux artistes «issus des milieux d'immigrants, des classes laborieuses et des minorités» de participer à l'exploration de l'esthétique de la spontanéité.

Je voudrais au contraire faire remarquer que les artistes issus des minorités qui, au début des années soixante, travaillaient dur pour se frayer un accès à la scène, ne pouvaient se permettre le privilège d'expérimenter en public. Même si elle se produisait avec en toile de fond le jazz, une tradition musicale extrêmement populaire et influente qui transcende les limites entre le "grand art» et le «divertissement», l'expérimentation de l'improvisation dans les performances, en théâtre et en danse, était essentiellement le fait d'artistes blancs. En examinant les fondements épistémologiques du jazz, où l'improvisation opère par une synthèse réalisée à partir d'oppositions binaires et agit non pas comme un moyen de s'abandonner complaisamment à son individualité mais comme une technique de construction de la démocratie, j'espère montrer le caractère artificiel de l'opposition entre la spontanéité et la structure. Je voudrais plutôt soutenir que l'altérité est rendue possible en partie grâce à la permanence de ces oppositions. Dans un deuxième temps, cet article s'intéressera aux interventions du premier féminisme dans un monde des arts visuels dominé par les hommes et proposant, par le fait même, une approche masculine de l'altérité, afin de mettre au jour la spécificité sexuelle des médiums artistiques. En chorégraphiant une sorte de pas de deux sur le rôle que jouent le sexe et la race de l'artiste dans sa façon d'envisager l'Autre, j'espère mettre au jour quelques-unes des figures sous lesquelles la danse a configuré cet Autre.

\section{DÉCOUVRIR DU NEUF}

Les expérimentations du théâtre d'avant-garde des années soixante, telles celles du Living Theater, de l'Open Theater et de Jerzy Grotowski, donnaient au 
corps un accès privilégié à la spontanéité au moyen d'un mélange de diverses pratiques physiques comme le yoga, les exercices de groupe, la psalmodie, le candomblé et le massage - toutes perçues comme des moyens de faire naître une meilleure prise de conscience des sensations et de se donner une corporéité poreuse et sensible. Les programmes d'entraînement mis sur pied par ces troupes de même que ceux que préconisait Viola Spolin, dont l'enseignement dans le domaine du jeu de l'acteur exerçait alors une grande influence, faisaient tous appel à des rencontres ouvertes entre les acteurs au cours desquelles ils expérimentaient la scène avec tout leur corps, regardant, reniflant, écoutant leur environnement et répondant par leurs mouvements aux sollicitations de cet environnement. Beaucoup de leurs performances produisaient des structures narratives à partir de l'exploration physique et des rencontres entre les corps. Au lieu de partir du texte d'une pièce, leurs dialogues provenaient souvent d'échanges spontanés entre les acteurs lors des répétitions. Les grognements, les cris, les plaintes et les soupirs exprimaient tous la façon dont les acteurs s'investissaient totalement dans l'instant et témoignaient de l'authenticité de leur accès aux couches les plus profondes de la sensation. Lors de la performance, les périodes de silence qui accompagnaient les mouvements du corps soulignaient encore plus cette volonté d'atteindre à la dimension brute, sans médiation, de l'expérience humaine considérée comme source d'inspiration potentielle de transformation sociale.

Dans le domaine de la danse, des expérimentations telles celles des chorégraphes de la Judson mettaient l'accent sur la dimension physique d'actions comme chuter, courir et se livrer à des pedestrian tasks plutôt que de traiter le corps comme signe d'une intériorité plus fondamentale ou plus profonde que les mots dont naissait l'action. Et pourtant, le désordre ludique célébré dans ces performances faisait avant tout du corps une voie d'accès privilégiée à des possibilités de perception susceptibles de donner lieu à de véritables découvertes. Témoignant en faveur du plaisir sérieux que l'improvisation pouvait procurer, la critique Jill Johnston l'analysait ainsi:

The demonstration of play as a mode of action central to dance is a significant regression. Central to play is improvisation. To improvise is to compose on the spur of the moment, as the dictionary says. Children compose things offhand in this manner and don't worry about alternative ways of behaving. When children like something they've done, they repeat it, thus achieving a set form, such as hopscotch or red-light. Adults who regress to childhood do the same... ${ }^{2}$

Johnston définit ici l'enfance comme un espace dans lequel l'exploration, menée au gré du hasard et dégagée de toute habitude, peut conduire à de nouvelles façons extraordinaires de se comporter. Engagés dans ces explorations indéterminées des capacités du corps, les artistes du groupe Judson envisageaient les diverses potentialités offertes par le corps comme une ouverture démocratique des possibles. Ils déhiérarchisaient les diverses parties du corps, accordant à la tête, au torse, au bassin, aux doigts, aux jambes ou aux coudes le même intérêt. L'espace et le lieu étaient également débarrassés de toutes les associations traditionnellement attachées à eux: c'est ainsi que le haut, le bas, le dedans et le dehors pouvaient être inversés, juxtaposés, entremêlés, par l'effet d'improvisations vouées à la perturbation des automatismes.

Le contact improvisation, développé par Steve Paxton et quelques autres, au début des années soixante-dix, poursuivait cette entreprise de déstabilisation des codes spatiaux et corporels en mettant l'accent sur la modification des points de contact entre deux corps. Encourageant les danseurs à ignorer la forme du corps, le rythme et le phrasé, le contact improvisation recherchait l'absorption dans le flux physique du toucher et l'élan donné par les poids combinés des corps lors du contact. Résolument désinvolte dans son approche, le contact improvisation lançait un défi aux protocoles de la danse par ses véritables jams de longueur indéterminée et ses multiples possibilités de contact corporel entre tous les participants. Il exploitait le corps comme une source d'immédiateté 
éternelle défiant toute forme d'enrégimentement ou d'habitude.

L'historienne de la danse et critique Sally Banes caractérise en termes succincts la notion d'improvisation qui prévalait alors:

In a way improvisation in its purest form vould call for no choreography... it stands in direct opposition to choreography... So in a way improvisation is very subversive... And in that subversive role that improvisation has in its purest sense, improvisation really does away with all the conventions that we know, that we recognize a dance work by. ${ }^{3}$

Banes oppose la spontanéité de l'improvisation au souci de structure de la chorégraphie. Elle prétend que la chorégraphie consiste à choisir judicieusement les conventions de représentation auxquelles faire appel pour formuler un message dansé. L'improvisation, au contraire, perd toute conscience de ces conventions au profit de la performance d'une anti-forme. Là où la chorégraphie table sur une certaine recherche de la structure et sur l'habileté, l'improvisation offre la possibilité d'échapper aux préoccupations formelles, ce qui lui permet de fonctionner de façon subversive, sinon pour détruire complètement les protocoles établis, du moins pour en dévier.

Pour avoir accès aux impulsions les plus spontanées, et donc les plus authentiques, de nombreux artistes ont jugé bon, comme l'a fait remarquer Banes, de se lancer dans des façons d'agir qui ne fassent appel ni au jugement ni à la conscience. De façon assez proche du naturel préconisé par la première génération des chorégraphes modernes, ils n'ont conçu la découverte que comme un abandon de toute les formes établies, de toutes les habiletés reconnues. Fondant leurs recherches sur la différence absolue entre penser et faire, ils s'efforçaient d'ouvrir un espace de nouveauté d'où surgirait une invention radicalement hors normes.

Stigmatisant cette tendance générale qu'elle qualifie de "thérapie, catharsis et même joyeux laisser-aller", la chorégraphe Trisha Brown, du groupe Judson, décrit au contraire son approche de l'improvisation de la façon suivante:
[...] if in the beginning you set a structure and decide to deal with $X, Y$, and $Z$ materials in a certain way, nail it down even further and say you can only walk forward, you cannot use your voice or you have to do 195 gestures before you hit the wall at the other end of the room, that is an imrprovisation with set boundaries. That is the principle, for example, behind jazz. The musicians may improvise, but they have a limitation in the structure just as improvisation in dance does. This is what I would call structured improvisation because it locates you in time and place with content. 4

Selon Brown c'est le fait d'imposer à l'improvisation certaines limites ou structures qui permet au performer de découvrir ou d'inventer de nouvelles choses. Dans une des rares références faites au jazz par un des artistes du groupe Judson, elle voyait là le point commun entre les deux pratiques artistiques en ce qui a trait au processus de création.

Le jazz inspirait alors aussi bien les artistes blancs que leurs confrères afro-américains et son influence se faisait particulièrement sentir, dans les années soixante, sur le théâtre et la danse. De nombreux artistes allaient régulièrement dans des clubs de jazz pour écouter la synthèse radicale entre le connu et l'inconnu que cette musique opère. Mais comment le jazz vivait-il, quant à lui, la relation entre l'habileté du musicien et sa spontanéité? Comment l'improvisation de jazz permettait-elle de concevoir la relation entre la pensée et l'action?

\section{STRUCTURER LA SPONTANÉITÉ}

Les musiciens de jazz se servent de leur compréhension individuelle des principes du rythme, de l'harmonie et de la mélodie comme d'une carte pour explorer de nouveaux territoires musicaux. Souvent basées sur des mélodies populaires ou sur des airs de blues, les improvisations de jazz se livrent à une recomposition continuelle d'un matériau déjà connu, une recomposition qui témoigne de l'inventivité individuelle et collective des musiciens. Remuante, aventureuse et toujours en quête de changement, la tradition du jazz a fait naitre des performances comme celle, en particulier, du trompettiste Louis Armstrong au début de sa carrière: 
Louis Armstrong would improvise on the same theme for a full half-hour, taking twenty choruses in a row... His imagination seemed inexhaustible; for each new chorus he had new ideas more beautiful than those he had reproduced for the preceding chorus. As he went on, his improvisation grew hotter, his style became more and more simple - until at the end there was nothing but the endless repetition of one fragment of melody - or even a single note insistently sounded and executed with cataclysmic intonations. ${ }^{5}$

Les innovations d'Armstrong, basées sur les structures harmoniques et rythmiques préétablies de la mélodie et utilisant le format que leur donnait le chorus, en arrivaient à produire un minimalisme radical, qui n'entretenait plus qu'un lointain rapport avec les phrases de départ. Et chaque nouveau chorus se fondait sur le chorus précédent pour faire surgir encore du nouveau.

Pour donner forme à la progression de ces solos, Armstrong, aussi bien que ceux qui l'écoutaient, devaient nécessairement occuper pleinement l'espace de l'ici-maintenant, d'où naissait le neuf, mais ils devaient aussi se souvenir du passé, c'est-à-dire en l'occurrence de chacun des choruses précédents avec lequel dialoguait toujours le chorus actuel, et ils accueillaient aussi le futur qui s'ouvrait devant eux, dans l'attente d'être composé. Tous les musiciens de l'orchestre participaient collectivement à cette histoire en marche de la composition, en écoutant attentivement les inventions d'Armstrong tout en sachant que la même possibilité allait bientôt s'offrir à eux.

Ainsi, non seulement le jazz oscille-t-il perpétuellement entre structure et immédiateté, forme et originalité, habileté et spontanéité, mais il offre aussi des possibilités exceptionnelles d'ajustement entre les besoins et les désirs individuels et collectifs. Les solos d'Armstrong prenaient place à l'intérieur de la structure partagée de solos pris alternativement par tous les membres de l'orchestre. Les expérimentations formelles ultérieures que connaitra le jazz avec le bebop et le free jazz des années soixante continueront de répartir collectivement la responsabilité de la création entre tous les membres de l'orchestre. Les musiciens de free jazz avaient beau ne plus se servir d'un air connu de tous ni même d'harmonies préétablies, ils n'en travaillaient pas moins à se donner pour chaque morceau une cohérence fondée sur la conscience constante qu'ils avaient du matériau sonore mis en place dès les premiers instants de l'interprétation.

Ainsi le jazz établissait-il une tradition de renouvellement perpétuel face aux exigences sclérosantes de la musique commerciale. Il proposait aussi une synthèse exemplaire entre l'innovation sans préméditation et la critique sociale. La force militante du be-bop, sa vitesse et ses harmonies rugueuses, l'agilité de ses découpages, semblables à des effets de montage, tout, en lui, exprimait une provocante opposition à l'oppression raciale. Sa capacité de faire surgir le non-prémédité éblouissait les auditeurs par les possibilités qu'elle offrait d'agir en dehors du système et contre lui. Comme l'explique le dramaturge, poète et activiste Amiri Baraka:

[...] the music was a feast to the rhythm-starved young white intellectuals as well as to those young Negroes, uncommitted to the dubious virtues of the white middle class, who were still capable of accepting emotion that came from outside the shoddy cornucopia of popular American culture. 6

Même le cool jazz qui a succédé au be-bop, avait une dimension non conformiste; il mettait en place une série de paramètres rythmiques et harmoniques axés sur l'exploration qui se démarquaient de façon spectaculaire de la culture qui continuait à produire des succès populaires du genre «Le petit chien dans la vitrine».

Étrangers au conformisme qui prévalait dans l'Amérique de l'après-guerre, les artistes étaient également marginalisés par la piètre estime et le statut inférieur dans lesquels on tenait leur profession. L'insistance mise sur les questions économiques et sur les problèmes qui se posaient dans ce domaine avait pour effet, comme l'observe Baraka, de décourager "completely any significant participation of the imaginative sensibility in the social, political, and economic affairs of the society»? Les artistes blancs, qui se sentaient aliénés par cette dévalorisation de 
leur vocation, s'identifiaient à la situation des artistes afro-américains dont l'art était façonné par la discrimination raciale qui les avait opprimés pendant des générations. Cette assimilation de certains Blancs aux Afro-Américains a créé de nouvelles alliances qui ont alimenté le mouvement des droits civiques et les gains importants qu'il a pu apporter aux Afro-

Américains sur le plan de ces droits, mais elle a également provoqué certains malentendus, en particulier quand les Blancs, oublieux des différences historiques de classe et de race, prenaient pour acquis que l'improvisation était, au fond, mise au service de la même révolte.

Baraka distingue les rôles joués par l'improvisation dans la culture blanche et dans la culture afroaméricaine au moyen d'une comparaison entre Bix Beiderbecke et Louis Armstrong:

The white middle-class boy from lowa was the product of a culture which could place Louis Armstrong, but could never understand him... [Beiderbecke] had an emotional life that, as it turned out, was based on his conscious or unconscious disappproval of most of the sacraments of his culture. On the other hand, Armstrong was, in terms of emotional archetypes, an honored priest of his culture - one of the most impressive products of his society. Armstrong was not rebelling against anything with his music. In fact, his music was one of the most beautiful refinements of Afro-American musical tradition, and it was immediately recognized as such by those Negroes who were not busy trying to pretend that they had issued from Beiderbecke's culture. The incredible irony of the situation was that both stood in similar places in the superstructure of American society: Beiderbecke, because of the isolation any deviation from mass culture imposed upon its bearer; and Armstrong, because of the socio-historical estrangement of the Negro from the rest of America. Nevertheless, the music the two made was as dissimilar as is possible within jazz. ${ }^{8}$

L'esthétique de la spontanéité à l'œuvre dans la musique d'Armstrong provenait d'une matrice culturelle qui accordait une valeur insigne à tout ce qui était improvisé, tandis que la culture de la classe moyenne blanche qui avait produit Beiderbecke condamnait l'improvisation. Beiderbecke évoluait donc à l'encontre des normes et des canons de sa culture alors qu'Armstrong, lui, se servait d'une tradition d'improvisation respectée, à la fois pour fortifier la culture afro-américaine et pour s'élever contre son histoire d'oppression raciale. L'héritage de cette oppression avait donné chez chacun des deux artistes des résultats très différents. C'est ainsi que, même si le jazz représentait une pratique esthéticopolitique capable à la fois d'offrir un refuge contre le courant dominant de la société blanche et de le critiquer, il ne signifiait pas le même genre de résistance dans chacune des deux cultures.

\section{L'ENGENDREMENT DE LA DÉCOUVERTE}

Le jazz offrait un modèle d'organisation politique collective qui mettait en évidence et en même temps contestait les oppositions sur lesquelles se fondait la pratique artistique d'avant-garde. La production artistique féministe, quant à elle, proposait une autre approche, une approche qui contredisait elle aussi les oppositions entre forme et informe, structure et chaos, individu et groupe. Mais la critique féministe qui émerge dans les années soixante met aussi en évidence la complexe politique sexuelle qui gouvernait les diverses pratiques artistiques. Que l'on pense un instant au fait que la tradition du jazz dont je viens de parler était presque exclusivement masculine. L'intervention des musiciens de jazz à l'intérieur de la musique, elle-même art masculin, se faisait au détriment des femmes musiciennes, à l'exception des chanteuses, le chant étant considéré comme une discipline féminine. Il convient dès lors de se demander comment, en ce qui a trait aux caractéristiques sexuelles attribuées aux disciplines artistiques, les artistes féministes ont pu offrir une alternative quant à la structuration épistémologique de ces catégories.

L'œuvre de Carolee Schneeman, artiste formée en arts visuels mais commençant à travailler dans le domaine de la performance au début des années soixante, en constitue un exemple capital. Des œuvres comme Newspaper Event (1962), Lateral Splay (1963), toutes deux données lors des concerts du groupe 
Judson, et Meat Joy (1964) donnent à la dimension tactile et sensible de la corporéité le sens d'un labeur commun. Introduisant le collage dans le temps et l'espace de la performance, elles exigent du corps des artistes qu'il s'engage dans des activités disparates mais entrecroisées dont la rencontre un peu rude donne son sens à la performance. Pour Newspaper Event, Schneeman a demandé à chacun des sept danseurs de se concentrer sur une partie du corps différente, un type d'action différent et une phrase parlée différente. Dans ce qui constituait le prélude à ces actions improvisées discrètes mais se chevauchant les unes les autres, les danseurs lançaient dans l'espace des piles de journaux, créant ainsi le milieu malléable, glissant et réactif dans lequel ils bougeaient. Pendant dix minutes, ils se pliaient, se roulaient, s'ébattaient et se laissaient tomber dans les feuilles de papier qu'ils déchiraient et lançaient aussi. Lateral Splay offrait le même genre de surabondance visuelle et kinesthésique, mais la composition de la pièce mettait en jeu une structure très différente. Douze danseurs devaient choisir parmi quatre types de courses, toutes menées avec «a maximum expenditure of directed energy " ${ }^{9}$. Ils devaient changer de course chaque fois qu'ils entraient en collision avec un autre danseur. Schneeman a travaillé longuement avec les danseurs les techniques de collision de façon à leur permettre d'acquérir les habiletés nécessaires à une bonne utilisation de la gravité et de l'élan. Le résultat, trois minutes explosives de trajectoires entrecroisées, d'impacts étourdissants et d'énergies frénétiques, fut qualifié par Jill Johnston de "the wildest dance on the program" (la danse la plus sauvage de tout le programme) du treizième concert des chorégraphes de la Judson. Meat Joy combinait de la peinture et du papier avec des corps humains presque nus, du poisson cru, des poulets plumés et des hot-dogs, traitant toutes les surfaces comme si elles avaient la même sensualité, la même capacité de communiquer par friction. Quand les seins, les bas-ventres, les aisselles, les ventres, les fesses, les cuisses, les pieds, les dos, les poitrines, les lèvres, les oreilles, les doigts, les cous se collaient et gigotaient les uns contre les autres, lubrifiés par la chair gluante de la viande, de la volaille et du poisson, les zones érogènes traditionnelles perdaient leur suprématie. C'est le corps tout entier qui devenait le site d'un jeu érotique.

Ces expérimentations étaient inspirées par la conviction de Schneeman que le pouvoir de l'art tient à ses excès. Elle écrit:

I have the sense that in learning, our best developments grow from works which initially strike us as "too much»; those which are intriguing, demanding, that lead us to experiences which we feel we cannot encompass, but which simultaneously provoke and encourage our efforts. Such works have the effect of containing more than we can assimilate; they maintain attraction and stimulation for our continuing attention. We persevere with that strange joy and agitation by which we sense unpredictable rewards from our relationship to them. These "rewards" put to question - as they enlarge and enrich correspondences we have already discovered between what we deeply feel and how our expressive life finds structure. 10

Schneeman décrit ici une pratique artistique qui invite les spectateurs à vivre une sorte de partenariat avec l'œuvre d'art dans son évolution. Elle conçoit la mission de l'art comme produisant une amplification mais aussi une intégration et un équilibre de nos sentiments et de la façon dont nous les structurons.

Pour pouvoir mener cette approche de l'art fondée sur la surenchère plutôt que sur l'alternative, Schneeman avait abandonné le monde des arts visuels pour celui de la performance, créant ce qu'elle appelait peinture cinétique ou actualisations, des performances au cours desquelles «the fundamental life of any material used is concretized " 11 . Pour Schneeman, le fait de privilégier le corps comme site de tout événement sensuel représentait une façon d'exposer les présupposés masculins sous-tendant l'expressionnisme «abstrait», tout en montrant en même temps de façon palpable ce à quoi pourrait ressembler l'" action painting ». Elle note :

In 1963 to use my body as an extension of my paintingconsciousness was to challenge and threaten the psychic territorial power lines by wich women were admitted to the ArtStudClub. ${ }^{12}$ 
Là où l'artiste mâle travaillait à sécuriser son moi, tenu à distance de l'œuvre d'art que par ailleurs il contrôlait, les performances de Schneeman catapultaient à la fois le performer et le spectateur dans un univers sensoriel à l'énergie renouvelée.

Comme l'a fait remarquer l'historienne de la danse Karen Schaffman, les efforts de Schneeman tombaient dans un vide entre deux disciplines artistiques dont aucune ne souhaitait la reconnaître pour sienne ${ }^{13}$. Excommuniée du monde des arts visuels pour cause de subjectivité érotisée dans un exhibitionnisme débridé, elle se trouvait tout autant boudée par les chorégraphes dont les efforts étaient tout entiers tournés vers la masculinisation du monde féminocentriste de la danse moderne. C'est ainsi que la critique Jill Johnston citée plus haut, chantant les vertus des jeux enfantins offerts lors des concerts de la Judson, stigmatise les contributions de Schneeman à ces concerts en les qualifiant de «messy, brainless, "happening" with lots of clothes, paper, rags, burlap, and paint» ou les considère comme "a wild orgy» 14 .

Les artistes de la Judson, dans leur revendication d'une approche non expressionniste de la recherche chorégraphique, adoptaient une esthétique largement stochastique en faisant appel à toute une variété de procédures aléatoires ou simplement en s'en remettant à ce qu'elles produisaient. Comme leur mentor, le compositeur John Cage, ils empruntaient énormément à la pensée zen et taoïste du hasard, se servant de ces formulations abstraites de l'ordre des choses pour déconstruire les méthodes de composition trop soucieuses d'intériorité. Bien que certains des artistes de la Judson aient souligné la similitude entre leurs recherches et la musique de jazz, les approches afro-américaines de l'improvisation restaient largement ignorées alors que les influences asiatiques se voyaient sans cesse célébrées.

Schneeman nommait cet usage de méthodes aléatoires «Fro-zen", défendant plutôt, quant à elle, une application alternative du hasard considérée comme "depth run on intent" (profondeur par intention), c'est-à-dire un moyen d'accéder à la nécessité du désir qui étend ses tentacules sous la surface des choses ${ }^{15}$. Bien qu'elle se produisît dans les concerts de la Judson, sa rhétorique de la surface et de la profondeur et ses excès intuitifs étaient en contradiction flagrante avec l'insistance mise sur la neutralité d'actions purement concrètes, position de principe avec laquelle ces artistes abordaient toutes les possibilités de mouvement, quelles qu'elles soient. Comme preuve de cette incompatibilité, Schaffman souligne les extraordinaires similitudes entre les recherches de Schneeman pour Lateral Splay et le développement, une décennie plus tard, du contact improvisation par Steve Paxton.

Schneeman avait inventé une forme d'écriture féminine ${ }^{16}$ pour la scène, en faisant appel à des préoccupations charnelles, traditionnellement considérées comme féminines, et l'utilisait comme antidote aux préceptes désincarnés de l'expressionnisme abstrait. Je voudrais ici examiner rapidement un autre exemple, pris dans l'œuvre de Shigeko Kubota, membre du collectif Fluxus de performers. Il s'agit d'une forme différente d'attaque féministe lancée contre le monde masculin de l'art. Dans Vagina Painting (1965), Kubota fixait un pinceau à ses sous-vêtements et le trempait à plusieurs reprises dans un pot de peinture rouge. Puis elle allait sur la pointe des pieds s'accroupir à divers endroits d'un plancher recouvert de papier et elle peignait ainsi. La façon précaire et pathétique dont son corps s'accroupissait pour tenter maladroitement de manœuvrer le pinceau montrait l'artiste dans un rôle avilissant. L'entrejambe, dépourvu de la coordination musculo-squelettique qui seule aurait pu permettre une telle manœuvre, restait passif; aucun niveau de compétence technique n'aurait pu permettre un meilleur contrôle du pinceau et le corps accroupi pouvait s'écrouler à n'importe quel moment. L'activité qui consistait à tremper le pinceau et à le faire courir sur le papier était ridicule mais aussi ironique. Elle faisait référence aux menstruations et à la fécondité, les traits propres aux femmes dans la sphère naturelle plutôt que dans celle de la culture, tout en symbolisant la précarité de la position des femmes dans le monde de l'art. L'œuvre présentait 
l'alternative «féminine» à l'action painting de Jackson Pollock et émettait même un commentaire sur les références qu'il faisait, dans sa découverte de la peinture spontanée, à des influences «orientalistes». Elle faisait également référence aux premières expérimentations du Gutai dans l'art de la performance, dans son Japon natal, expériences qui avaient déjà considérablement élargi le concept d'action painting 17 .

Là où Schneeman privilégiait une surabondance de sensations, Kubota propose un commentaire ironique à niveaux multiples. Mais les deux utilisent la performance comme une force permettant de s'attaquer à la domination masculine du monde des arts visuels et les deux se servent de l'improvisation comme d'un outil permettant de faire surgir de nouvelles formes de connaissance inscrites dans le corps. Du point de vue du jazz, les performances de Schneeman comme celles de Kubota négligent d'établir une cohésion structurelle négociée en commun pendant toute la durée de la pièce. Les deux se fondent sur les prémisses d'une altérité critique et engagée mais leurs performances ne fournissent pas les moyens de transformer cette altérité en socialité 18 . Il reste, par ailleurs, que d'un point de vue féministe, le jazz pratique une exclusion fondée sur le sexe, sinon en théorie, du moins en pratique.

\section{IMPROVISER LES AUTRES}

Au cours des dernières années, la catégorie de l'Autre, longtemps conçu comme une altérité unique et singulière, s'est manifestement incarnée de façon fort différente selon les contextes historiques et sociaux. Dans son essai intitulé "The Other Question", Homi Bhabha fait remonter les effets du discours colonialiste et de sa construction de l'altérité à une confiance et un investissement dans les catégories oppositionnelles de l'expérience. L'Autre, explique Bhabha, tire son existence non de la convocation des attributs de l'altérité, mais du vacillement entre des valeurs binaires représentant le Soi et l'Autre. C'est la rigidité de catégories oppositionnelles, telles que l'ordre et le désordre, la civilisation et l'état sauvage, la censure et le désir, l'ordre et l'anarchie, et un investissement ambivalent des deux côtés des couples d'oppositions qui perpétuent le discours colonialiste sur l'altérité. Dans son étude des pratiques coloniales victoriennes, intitulée Imperial Leather, Anne McLintock étend et raffine l'analyse de Bhabha en analysant la dynamique complexe à travers laquelle le discours colonialiste exerce sa domination selon des clivages liés au genre, à la race, à la classe et au sexe. Elle montre comment ces catégories, se projetant souvent les unes sur les autres, apparaissent toujours liées intimement les unes aux autres.

Ce que les exemples du jazz afro-américain et de la performance féministe révèlent, c'est la façon dont ces catégories oppositionnelles opéraient dans les performances des années soixante. Ils font aussi voir la nécessité qu'il y a de considérer les façons différentes dont les discours sur l'altérité se constituent dans les différents médiums et pratiques artistiques. Par exemple, les expérimentations théâtrales du début des années soixante, telles celles du Living Theater et de l'Open Theater, remettaient en jeu la priorité accordée au texte de la pièce, considéré comme masculin, par rapport à celle de l'action, considérée comme féminine, en privilégiant les découvertes faites par le corps lors de l'improvisation, que ce soit lors des répétitions ou pendant la représentation elle-même. Elles maintenaient cependant l'opposition sous-jacente entre le texte et le corps en concevant le corps comme la source d'une spontanéité non médiatisée par l'habileté technique. Le corps servait d'emblème à la frontière psychosomatique que l'on pouvait explorer pour y faire surgir de nouvelles découvertes. Puisant dans ce territoire inconnu, les performers revenaient au texte de la pièce, rajeunis et revitalisés. Ils maintenaient ainsi une attitude paternaliste dans le théâtre d'avant-garde.

Les artistes de la Judson, eux, contestaient ces oppositions en plaçant le texte et le corps, l'intérieur et l'extérieur sur la même surface plane. Pourtant, de nombreuses personnes qui assistaient à ces performances, dont la critique Jill Johnston, ne 
parvinrent à interpréter l'usage que faisaient les chorégraphes de l'improvisation qu'en termes de structures binaires. Comme le montre la description de Johnston citée ci-dessus, chacun des performers qui opère un retour brusque à l'enfance se montre individuellement capable de nouvelles découvertes exceptionnelles, mais le concept de régression ne fournit aucun modèle pour une collaboration collective dans la définition des règles de la performance. Individuelle plus que sociale, la formulation de structures de jeux enfantins aussi sophistiqués que la marelle provient simplement, si l'on en croit Johnston, de la répétition d'une action agréable. Le fait que la performance établisse un contrat social avec les autres ou que les jeux euxmêmes naissent de moments culturels spécifiques n'est pas pris en compte. Ce qui compte, c'est le moment individuel de la découverte au cours duquel des schèmes de perception convenus cèdent la place à une appréhension plus vitale de soi-même et de son environnement.

L'interprétation de Johnston repose sur une incapacité de transcender les catégories de la réflexion et de l'action et de penser un entre-deux.

Semblablement, la chorégraphe du groupe Judson, Elaine Summers, réalisant qu'elle vivait en fonction d'un schéma énergétique et d'une image du corps qui n'étaient pas les siens, décrit sa quête d'une façon originaire de se mouvoir en ces termes:

I spent a lot of time by myself doing that work, and one summer I felt, "If I'm really honest about it, all I want to do is lie on the floor. I really don't want to move». So for two-and-a-half months, during the three-hour period I spent dancing every day, I didn't get up off the floor. And in that time, I began to rediscover my own energy. 19

Summers décrit un processus qui consiste à oublier tout son entraînement antérieur, en restant simplement étendue au sol et en attendant que son corps entreprenne une suite d'actions. La nouvelle conscience qu'elle prenait de l'énergie qui finissait par émerger changeait radicalement, prétend Summers, sa perception du mouvement et sa relation à l'environnement. Plutôt que de prendre conscience de sa façon d'échanger avec les autres certaines habiletés physiques et perceptuelles, Summers assiste à l'émergence de sa propre énergie, dans ce qu'elle a d'unique et de neuf.

Avec sa formation en danse, la quête des schémas de sa propre énergie menée par Summers peut fort bien avoir été guidée par des impulsions féministes qui la poussaient à reprendre possession d'un corps dominé par des techniques d'entraînement et une esthétique plus masculines. Et pourtant, en même temps, sa situation dans le champ de la danse, médium artistique féminisé, donnait à sa recherche le même genre de féminité excessive que celle qui rendait le travail de Schneeman inefficace. Si, au lieu de penser son projet comme une façon de découvrir sa propre énergie, elle avait pu le concevoir comme un moyen d'acquérir des techniques nouvelles et différentes, elle aurait pu dépasser les oppositions qui maintenaient la danse à sa place.

Opposer le chorégraphié, perçu comme l'habituel, et l'improvisé, envisagé comme le spontané, revient à méconnaître la capacité qu'a le corps de prendre des décisions fondées sur l'entraînement et sur la façon dont il peut ruser avec les attentes habituelles. Qu'on les conçoive comme les «techniques du corps» de Marcel Mauss ou comme formant l'«habitus» dont parle Pierre Bourdieu, les systèmes de coordination qui constituent les moyens d'expression d'un performer forment l'armature à travers laquelle se produisent explorations et découvertes. Comme le jazz le démontre très clairement, les interprètes découvrent des formulations musicales jusque-là inconcevables en modifiant les techniques qu'ils possèdent déjà. Et cette façon de découvrir du nouveau concerne à la fois les moments individuels de la performance et la structure d'ensemble ainsi que l'organisation d'un morceau.

Même les premiers improvisateurs de danse contact, dont les jam-sessions faisaient voler en éclats tous les formalismes de la scène et ses buts expressifs, n'en élaboraient pas moins une perspective chorégraphique dans l'organisation et l'exécution de leurs performances. Bien qu'ils prétendissent se 
défaire de l'habitude pour se brancher sur le primal, leur pratique peut fort bien être considérée comme une façon de repositionner et de redéfinir une semiosis chorégraphique. Ils se préoccupaient en effet manifestement de leur orientation dans l'espace, de leur localisation dans l'espace, de la synchronisation et de la qualité des diverses énergies mises en jeu, de leur persévérance dans une action donnée ou dans la danse avec une autre personne. Et en se préoccupant de tout cela, ils entraient nécessairement en conflit avec les conventions de la représentation qui, dans la danse, régissent la construction du sens.

\section{IMPROVISER LA CHORÉGRAPHIE}

$\mathrm{Si}$, comme le prétend Bhabha, l'altérité dépend de la fixité de catégories comme la structure et la spontanéité, la forme et l'informel, ou l'intelligence et l'instinct, alors les exemples du jazz et de la performance féministe offrent des modèles d'improvisation qui pourraient bien nous faire dépasser le projet de l'appréhension de l'Autre. Mais Bhabha situe la possibilité d'échapper au discours colonialiste dans les potentialités de l'imitation, une performance pratiquée par l'Autre de l'investissement colonialiste situé des deux côtés des catégories oppositionnelles, alors que le jazz et la performance féministe contestent et dépassent l'obligation des oppositions binaires elles-mêmes. Chacune de ces pratiques propose, à sa façon, une critique sociale des relations de pouvoir et la formulation d'une façon de contester les différentiels de pouvoir qui se créent lors de la construction de l'altérité. Ensemble, elles attirent notre attention sur les structurations complexes du pouvoir à travers lesquelles se perpétuent les discours de l'altérité. Si on considère le couple qu'elles forment, il peut devenir possible d'envisager de nouvelles coalitions entre les artistes déçus des divers discours sur l'altérité, des coalitions qui pourraient nous mener à un nouvel activisme social, démocratique et égalitaire.

C'est pourquoi Belgrad et Banes ont raison d'insister sur le potentiel subversif de l'improvisation. Mais, comme j'ai tenté de le montrer ici, la dimension subversive du jazz propose, plutôt qu'un abandon de toute structure, une nouvelle vision de la relation de l'individu et du groupe, et du rapport entre l'expérience de la liberté et la discipline. En regardant des performers et des musiciens de talent qui travaillent ensemble, s'appuyant mutuellement dans leurs initiatives, les spectateurs acquièrent de façon palpable le sentiment qu'une organisation alternative de la vie politique et sociale est possible. La liberté, et non ce qui s'atteint dans l'absence de toute structure, dépend de l'usage que font de la structure toutes les personnes engagées dans un projet donné. Baraka attribue au jazz, avec la dimension collective qu'il donne à l'action spontanée, le rôle d'avoir aidé à frayer le chemin emprunté par les anciens esclaves dans leur accession à la citoyenneté pleine et entière. Méprisant la définition que donnaient de la démocratie des années cinquante marquées par le maccarthysme en l'assimilant à l'anti-communisme, Baraka imagine une citoyenneté dans laquelle la liberté individuelle et la cohésion du groupe seraient complémentaires. Le petit ensemble de jazz, considéré comme pouvant servir de modèle à l'organisation politique, réaffirme la différence individuelle dans l'instant même où il consolide et renforce la conscience sociale.

Aussi inspirant qu'ait pu être le jazz pour les artistes des années soixante, aussi vigoureusement qu'il ait pu remettre en question les oppositions binaires à travers lesquelles on crée l'altérité, une application contemporaine de ses principes exige une reconnaissance encore plus grande de la différence, une reconnaissance susceptible d'inclure aussi bien le sexe et les préférences sexuelles que la race et la classe. Les performances féministes des années soixante en ont clairement montré la nécessité. Leur exploration de matériaux différents et de méthodes de compositions alternatives ont démasqué les privilèges dévolus aux hommes dans le monde de l'art. Leurs performances représentaient autant de commentaires sur tout le processus avant-gardiste de la découverte et de la domination de l'Autre par lequel l'artiste mâle assurait son statut de membre d'une élite. Donnant à cette altérité une voix critique puissante, elles parlaient 
haut et fort, célébrant un ensemble de valeurs exclusivement féminines et féministes. Mais les succès divers remportés par leurs interventions à l'intérieur de pratiques artistiques différentes nous rappellent les spécificités qu'il faut reconnaître à chaque médium.

De façon assez intrigante, ce que le jazz et la performance féministe partagent, c'est une tradition de décisions prises en commun dans le processus créatif de la production artistique. En s'appuyant sur leurs approches de la négociation consensuelle, il devrait être possible de chorégraphier, en toute spontanéité, une rencontre entre ces deux formes d'art qui soit aussi une commotion susceptible de mettre à jour les stratégies de l'altérité qui prévalent et de produire une série d'interventions improvisées qui éroderaient profondément les fondements de leur pouvoir. En pratiquant une approche de la danse aussi multivalente et multiplicatrice, on pourrait fort bien prendre conscience de la possibilité pour des corps, pensant spontanément et agissant de façon réfléchie, de s'engager collectivement dans la célébration, non pas de l'altérité, mais de la différence.

\section{N O TES}

1. D. Belgrad, The Culture of Spontaneity: Improvisation and the Arts in Postwar America, Chicago, University of Chicago Press, 1998, p. 16. [Les formes " ouvertes " ou " hétéroglossiques " produites par l'avant-garde des années de guerre défiaientle pouvoir social de la tradition angloaméricaine dominante. L'esthétique de la spontanéité mettait davantage l'accent sur "l'honnêteté ", «la prise de conscience» et "l'authenticité " que sur la maîtrise des formes et des techniques traditionnelles préconisée par les institutions établies de la culture d'élite. Elle offrait ainsi une possibilité de conquête de l'autorité culturelle plus accessible aux aspirants issus de l'immigration, des classes laborieuses et des minorités.]

2. J. Johnston, "Dance Journal: Play", The Village Voice, 18 novembre 1965, p. 15 et 22. [Le fait de mettre en évidence le jeu comme une modalité de l'action essentielle à la danse représente une régression significative. Ce qui est essentiel au jeu, c'est l'improvisation. Improviser revient à composer sous l'inspiration du moment, comme le dit le dictionnaire. C'est de cette façon que les enfants composent spontanément diverses choses; ils ne se soucient pas de trouver d'autres façons d'agir. Quand les enfants aiment ce qu'ils ont fait, ils le répètent, créant ainsi une forme, comme la marelle et d'autres jeux semblables. Les adultes qui retournent à l'enfance font la même chose.]

3. "Panel Discussion on Improvisation ", printemps 1980, organisé par le University-wide Committee on the Arts, S.U.N.Y. Purchase. [D'une certaine façon, l'improvisation, dans sa forme la plus pure, n'aurait guère besoin de chorégraphie... elle s'oppose même directement à la chorégraphie... Alors, d'une certaine façon, l'improvisation est une pratique très subversive... Et dans ce rôle subversif qui est le sien, l'improvisation, dans son sens le plus pur, se débarrasse vraiment de toutes nos conventions, ces conventions grâce auxquelles nous reconnaissons une œuvre comme de la danse.]

4. Bien qu'écrivant cela en 1978, Brown rattache cette approche de l'improvisation à ses premières œuvres avec A. Halprin et à des expérimentations subséquentes avec S. Forti. Voir A. Livet, Contemporary Dance: An Anthology of Lectures, Interviews and Essays with Many of the Most Important Contemporary American Choreographers, Scholars and Critics, New York, Abbeville Press, 1978, p. 44-45. [... quand dès le début vous vous imposez une structure en décidant de faire appel à des matériaux X, Y et Z, que vous traitez d'une certaine façon, et si vous enfoncez encore plus le clou en décidant, par exemple, que vous ne pourrez vous déplacer que vers l'avant, que vous ne devrez pas vous servir de votre voix ou que vous devrez faire 195 gestes avant d'atteindre le mur qui est à l'autre bout de la pièce, il s'agit d'une improvisation aux frontières délimitées à l'avance. C'est le principe que l'on retrouve, par exemple, derrière le jazz. Les musiciens peuvent improviser mais ils ont une limite qui leur est imposée par la structure, exactement comme pour l'improvisation en danse. C'est ce que j'appellerais une improvisation structurée parce qu'elle vous situe dans le temps et l'espace en vous donnant un contenu.]

5. D. Bailey, Improvisation: Its Nature and Practice in Music, London, British Library National Sound Archives, 1992, p. 50. [Louis Armstrong improvisait sur le même thème pendant toute une demi-heure, prenant jusqu'à vingt choruses d'affilée. Son imagination semblait inépuisable ; à chaque nouveau chorus il apportait de nouvelles idées, encore plus magnifiques que celles qu'il avait formulées dans le chorus précédent. Plus il jouait, plus son improvisation devenait intense et plus son style se simplifiait - jusqu'à ce qu'à la fin il ne restât plus que la répétition sans fin d'un fragment de mélodie ou même d'une seule note poussée avec insistance et formulée avec des intonations cataclysmiques.] 
6. I. Amiri Baraka [Leroi Jones], Blues People: Negro Music in White America, New York, William Morrow \& Company, 1963, p.200. [... la musique était une véritable fête pour les jeunes intellectuels blancs avides de rythme comme pour ceux des jeunes Noirs qui, restés imperméables aux douteuses vertus de la classe moyenne blanche, étaient encore capables d'accepter une émotion venue de l'extérieur du médiocre tout-venant de la culture populaire américaine.]

7. Ibid., p.230. [complètement toute participation significative des gens doués d'une imagination et d'une sensibilité artistiques aux dimensions sociales, politiques et économiques de la société.] 8. Ibid., p. 153-154. [Le jeune homme issu de la classe moyenne de l'Iowa était le produit d'une culture qui pouvait situer Louis Armstrong mais ne parviendrait jamais à le comprendre... [Beiderbecke] avait une vie émotionnelle qui, comme on a pu le voir, se fondait sur un rejet conscient ou inconscient de la plupart des valeurs consacrées de sa culture. Armstrong, au contraire, était, en termes d'archétypes émotionnels, un des grands prêtres les plus respectés de sa culture, un des produits les plus remarquables de sa société. Avec sa musique, Armstrong ne se révoltait contre rien. En fait, sa musique représentait au contraire un des plus magnifiques aboutissements de la tradition musicale afro-américaine et elle était immédiatement reconnue comme telle par ceux des Noirs qui ne passaient pas leur temps à essayer de prétendre qu'ils provenaient de la même culture que Beiderbecke. L'incroyable ironie de la situation c'est que tous les deux occupaient des places semblables dans la superstructure de la société américaine: Beiderbecke, à cause de l'isolement que toute déviation de la culture de masse imposait à ceux qui s'en rendaient coupables; et Armstrong, à cause de la séparation historique des Noirs du reste de la société américaine. Cela n'empêchait pas la musique que chacun d'eux faisait d'être aussi différente que possible à l'intérieur des cadres du jazz.] 9. C. Schneeman, More than Meat Joy, New Paltz, New York, Documentext, 1979, p. 47. [une dépense maximale d'énergie dirigée.] 10. Ibid., p. 9. [J'ai le sentiment qu'en apprenant, nous tirons le meilleur de notre développement d'œuvres qui nous frappent d'abord comme "excessives", celles qui nous intriguent, s'avèrent exigeantes et nous conduisent à des expériences que nous croyons ne pas pouvoir supporter mais qui en même temps provoquent et stimulent nos efforts. De telles œuvres ont pour principale caractéristique de contenir plus de choses que nous ne pouvons en assimiler; elles retiennent indéfiniment notre attention, par l'attirance qu'elles exercent sur nous et la stimulation qu'elles nous donnent. Nous persévérons donc, avec cette étrange joie, cette étrange agitation qui nous font sentir que la relation que nous entretenons avec elles nous réserve des récompenses imprévisibles. Ces « récompenses» remettent en question, en les élargissant et en les enrichissant, les correspondances que nous avions déjà découvertes entre ce que nous ressentons au plus profond de nousmêmes et la façon dont notre vie expressive trouve sa structuration.] 11. Ibid., p.9. [la vie fondamentale de tout matériau se trouve actualisée.]

12. Cité dans A. Jones, Body Art: Performing the Subject, Minneapolis, University of Minnesota Press, 1998, p. 3. [En 1963, me servir de mon corps comme d'une extension de ma conscience de peintre, c'était défier et même menacer les limites territoriales psychiques par lesquelles les femmes devaient passer pour être admises dans le Club Mâle des Artistes.]

13. K. Schaffman, «Excavating the Dinosaurs: Carolee Schneemann and Prehistoric Contact Improvisation ", communication présentée au Congress on Research in Dance, 3 décembre 1999.

14. La première citation décrit Chromelodeon et la seconde s'applique à Newspaper Event. Cité dans S. Banes, Democracy's Body: Judson Dance Theater, 1962-1964, Durham, Duke University Press, 1993, p. 149 et 96. [un happening sale et sans esprit, plein de vêtements, de papier, de chiffons, de sacs de jute et de peinture], [une orgie sauvage.]

15. Ibid., p. 15.

16. En français dans le texte (N.D.T.)

17. M. Kirby remarque que le groupe Gutai pourrait bien avoir joué un rôle capital dans l'invention des happenings. Voir Kirby, Happenings, New York, E.P. Dutton, 1965, p. 28-29.

18. Soit dit en passant, on pour ra trouver les schèmes d'une telle socialité une fois que le mouvement féministe aura vraiment pris son essor, par exemple, dans la Dinner Party (1979) de Judy Chicago, qui, en tant qu'événement à organiser, exigea un travail en commun s'étendant sur une période de temps significative.

19. E. Summers, cité dans E. Webb, "Teaching from the Inside Out", Soho Weekly News, 2 août 1979, p. 51. [J'ai passé énormément de temps seule, à faire ce travail, et un été, je me suis dit: «pour être tout à fait honnête, tout ce que je veux, c'est me coucher sur le sol. Je ne veux vraiment pas bouger $\%$. Alors, pendant deux mois et demi, pendant les trois heures que je passais chaque jour à danser, je ne décollais jamais du sol. Et c'est ainsi que j'ai commencé à redécouvrir ma propre énergie.] 